\title{
On the Structure and Complexity of Infinite Sets with Minimal Perfect Hash Functions
}

\author{
Judy Goldsmith ${ }^{*} \quad$ Lane A. Hemachandra ${ }^{\dagger} \quad$ Kenneth Kunen ${ }^{\ddagger}$
}

\begin{abstract}
This paper studies the class of infinite sets that have minimal perfect hash functionsone-to-one onto maps between the sets and $\Sigma^{*}$-computable in polynomial time. We show that all standard NP-complete sets have polynomial-time computable minimal perfect hash functions, and give a structural condition sufficient to ensure that all infinite NP sets have polynomial-time computable minimal perfect hash functions: If $E=\Sigma_{2}^{E}$, then all infinite NP sets have polynomial-time computable minimal perfect hash functions. On the other hand, we present evidence that some infinite NP sets, and indeed some infinite $P$ sets, do not have polynomial-time computable minimal perfect hash functions: if an infinite NP set $A$ has polynomial-time computable perfect minimal hash functions, then $A$ has an infinite sparse NP subset, yet we construct a relativized world in which some infinite NP sets lack infinite sparse NP subsets. This world is built upon a result that is of interest in its own right; we determine optimally - with respect to any relativizable proof technique--the complexity of the easiest infinite sparse subsets that infinite P sets are guaranteed to have.
\end{abstract}

\section{Introduction}

Algorithmic and complexity-theoretic approaches have, often separately, shaped theoretical computer science. This paper seeks a synergistic union of these two foundational research paradigms, following the emerging paradigm (surveyed in [Hem90]) of studying which classes of sets have efficient algorithms for operations other than membership. We study the class of infinite sets that have efficiently computable perfect hashing algorithms.

Hash tables are one of the most important data structures in use today, because of their time efficiency. There are several measures of the complexity of a given hash function: the size of the table relative to the size of the set being hashed, the expected number of collisions, the collision resolution algorithm, and the time and space used by the hash function. Additionally, if the hash function is being constructed algorithmically from the set to be hashed, one can consider the complexity of

\footnotetext{
-Department of Mathematics and Computer Science, Dartmouth College, Hanover, NH 03755.

tDepartment of Computer Science, University of Rochester, Rochester, NY 14627. Research supported in part by a Hewlett-Packard Corporation equipment grant and by the National Science Foundation under grant CCR8809174/CCR-8996198 and a Presidential Young Investigator A ward.

$\ddagger$ Department of Computer Sciences, University of Wisconsin, Madison, WI 53706. Research supported by the National Science Foundation under grant DMS-8501521.
} 
choosing or generating the function. In seeking a balance between these many measures, tradeoffs of various sorts are encounted [MNT90].

A hash function that has no collisions is called a perfect hash function [Knu73]; a perfect hash function, for a finite set $A$, that minimizes the size of the hash table (i.e., that uses a table of $|A|$ entries) is called a minimal perfect hash function [Spr77]. One approach to perfect hashing is to find a dynamic perfect hashing scheme, so the hash function changes as $A$ changes. Aho and Lee gave one such construction [AL88], and more recently Dietzfelbinger, Karlin, Mehlhorn, Meyer auf der Heide, Rohnert, and Tarjan [DKM ${ }^{+88}$ ] gave a probabilistic algorithm for dynamic perfect hashing. The present paper is devoted to the more demanding model of static hash functions, in which a single function (oblivious to the order in which various hashings are performed) must hash a given (infinite) set.

One can judge the space efficiency of a hash function (for a set $A$ ) by two in some sense dual measures: the number of bits needed to code the first $k$ strings of $A$, and the total number of bits of the strings in $A$ that map to the first $k$ strings in the target space. The first measure has been widely studied recently. In particular, an optimal minimal perfect hash function under this measure is the ranking function, $r_{A}$, which maps the $n^{\text {th }}$ string of $A$ to the $n^{\text {th }}$ string of $\Sigma^{*}$. Chang gave a generating scheme for ranking functions for finite sets based on the Chinese remainder theorem [Cha84]; Chang and Chang gave a simpler scheme, using a one-parameter function similar to a linear congruential generator [CC88]. Gordon [Gor89] gave a mathematical formulation for dynamic ranking of finite sets. However, in the present paper we are concerned with minimal perfect hash functions for infinite sets-one-to-one onto maps between the sets and $\Sigma^{*}$-and, unfortunately, recent work on ranking functions for infinite sets has shown that even extremely simple sets are unlikely to be polynomial-time rankable [GS85,Huy88,HR90]. Furthermore, under the second of the measures, there are no optimal minimal perfect hash functions, though ranking is pessimal. For these reasons-the pessimality of ranking and, more crucially, the provable complexity of ranking infinite sets-we are motivated to study minimal perfect hash functions that do not necessarily preserve lexicographic order.

We introduce the notion of minimal perfect hash functions on infinite sets by direct analogy to the finite case. A set $A \subseteq \Sigma^{*}$ has minimal perfect hash function $f$ if $f$ is a total function from $\Sigma^{*}$ to $\Sigma^{*}$ that, when viewed as a map from $A$ to $\Sigma^{*}$ is one-to-one and onto-that is, $f(A)=\Sigma^{*}$ and $(\forall a, b \in A)[f(a) \neq f(b)]$. Such functions are perfect in the sense that no two elements in the set being hashed collide, and are minimal in the sense that there are no unused addresses in the target space: viewed as a map between the set $A$ being hashed and $\Sigma^{*}$, these functions are bijections. However, though the minimality requirement assures that there are no gaps in the target space, it nonetheless is possible that a given minimal perfect hash function will fail to compress certain strings-and, in return, will have to compress others all the more dramatically in order to maintain minimality.

Though we briefly mention the quite straightforward case of recursion-theoretic (recursive) minimal perfect hash functions, we pursue in depth the more subtly nuanced study of complexitytheoretic (polynomial-time) minimal perfect hash functions. Section 3.1 explores the relationship between polynomial-time minimal perfect hash functions and sets having polynomial-time sparse subsets. There are simple conditions on sparse subsets of recursive sets that guarantee that those sets have polynomial-time minimal perfect hash functions, though there are unlikely consequences if all infinite sets in NP meet these conditions. In particular, Section 3.1 proves the following results. 
1. All standard NP-complete sets have polynomial-time minimal perfect hash functions.

2. If $\mathrm{E}=\Sigma_{2}^{E}$, then every infinite set in NP has a polynomial-time minimal perfect hash function.

3. If the exponential hierarchy collapses to exponential time, then each infinite set in the polynomial hierarchy has a polynomial-time minimal perfect hash function.

4. If all infinite NP sets have polynomial-time minimal perfect hash functions, then all infinite NP sets have infinite sparse NP subsets.

We present relativized evidence that the converse of part 2 above may fail, and we also construct an oracle relative to which there is an infinite set in $P$ (and thus NP) with no polynomial-time minimal perfect hash functions. Underlying this result is the establishment of absolute upper and relativized lower bounds on the subset structure of $P$ sets: though we note that all infinite $P$ sets have infinite sparse coNP subsets, our oracle shows that there are relativized worlds with infinite $P$ sets that have no infinite sparse NP subsets.

Section 3.2 considers polynomially honest polynomial-time minimal perfect hash functions for sets in the polynomial-time hierarchy. We prove that all high sets [Sch83] (and thus all complete sets for the $\Sigma$ levels of the polynomial hierarchy) lack such hash functions unless the polynomial hierarchy collapses.

Section 3.3 compares the sets compressible via polynomial-time minimal perfect hash functions and the sets compressible via polynomial-time ranking functions. We conclude that minimal perfect hashing is almost certainly a strictly broader notion than ranking: unless the polynomial hierarchy collapses, there are sets in $\mathrm{P}$ that are polynomial-time hashable but not polynomial-time rankable.

\section{Definitions}

$\mathrm{E}$ denotes the class $\mathrm{U}_{c}$ DTIME[2 $2^{\mathrm{cn}}$; EH denotes the exponential hierarchy [HIS85]. A set $A$ is in the class UP if it is recognized by a nondeterministic polynomial-time Turing machine that on no input has more than one accepting computation [Val76]. A set $A$ is in the class $D^{P}$ if there exist sets $B$ and $C$ in NP such that $A=B-C$ [PY84]. A total function $f$ is in the class \#P if there is a nondeterministic polynomial-time Turing machine $M$ such that for each $x, f(x)$ is the number of accepting computations of $M(x)$ [Val79]. $|x|$ denotes the length of string $x$.

Recall that a set $A \subseteq \Sigma^{*}$ is said to have minimal perfect hash function $f$ if $f$ is a total function from $\Sigma^{*}$ to $\Sigma^{*}$ that, when viewed as a map from $A$ to $\Sigma^{*}$, is one-to-one and onto- that is, $f(A)=\Sigma^{*}$ and $(\forall a, b \in A)[f(a) \neq f(b)]$. A set $A$ with polynomial-time minimal perfect hash functions is called $P$-compressible. We'll say that NP is $\mathrm{P}$-compressible ( $\mathrm{P}$ is $\mathrm{P}$-compressible) if all infinite sets in NP (P) are P-compressible. A function $f: \Sigma^{*} \rightarrow \Sigma^{*}$ is polynomially honest if there is polynomial function $h(n)$ such that for all $x \in \Sigma^{*},|x| \leq h(|f(x)|)$. If $h(n)$ is an increasing recursive function, then $f$ is recursively honest.

A set $A$ is $P$-rankable [GS85] if the function $r_{A}(x)$, the number of elements in $A$ that are lexicographically less than or equal to $x$, is polynomial-time computable. We call $r_{A}(x)$ the rank of $x$ in A.

A set $S$ is $P$-printable if there is a polynomial-time Turing machine that, on input $0^{n}$, outputs all strings in $S$ of length at most $n$ ([HY84], see also [Adl79,AR88]). A P-printable set is necessarily 
sparse and in P. (A set $S$ is sparse if the number of elements of $S$ of length at most $n$ is polynomial in $n$.)

A total function $f$ is in $\mathrm{NP}_{\mathbf{s v}}$, tot (NP, single-valued, total) if it is computed by a nondeterministic polynomial-time Turing machine such that for all $x$ : on input $x$, each computation outputs either $f(x)$ or $\perp$ (a symbol not in $\Sigma^{*}$ ), and at least one computation outputs $f(x)$.

Let $Q_{1}, Q_{2}, \ldots$ be a standard enumeration of nondeterministic polynomial-time Turing machines. Let $P_{1}, P_{2}, \ldots$ be a standard enumeration of deterministic polynomial-time Turing machines. Without loss of generality, we assume that $Q_{i}^{A}$ runs in nondeterministic time $n^{i}+i$, regardless of the oracle $A$, and that $P_{i}^{A}$ runs in deterministic time $n^{i}+i$, regardless of the oracle $A$.

\section{Results}

We study the class of sets having polynomial-time computable minimal perfect hash functions-the P-compressible sets. We demonstrate the P-compressibility of all standard NP-complete sets, give structural conditions sufficient to ensure the P-compressibility of all infinite NP sets, and give structural conditions necessary to ensure the P-compressibility of all infinite NP sets. We give essentially optimal bounds on the sparse subset structure of $P$. Finally, we note that, unless the polynomial hierarchy collapses, $\mathrm{P}$ contains $\mathrm{P}$-compressible sets that are not P-rankable.

\subsection{Sparse Subsets}

One of the major research themes in computational complexity theory (nicely surveyed in [Mah86]) is the investigation of sparse sets within complexity classes. We show that the existence of polynomialtime computable minimal perfect hash functions for a set $A$ is intimately related to the complexity of sparse subsets of $A$. Our first observation tells us that certain subclasses of $\mathrm{P}$ and NP have polynomial-time minimal perfect hash functions. An infinite set $B$ is well-spaced if all elements are exponentially spaced: $(x, y \in B, x \neq y) \Rightarrow \operatorname{abs}(|x|-|y|) \geq 2^{\min (|x|,|y|)}$, where abs( $)$ denotes the absolute value function.

Theorem 3.1 Any set in $\mathrm{E}$ that has an infinite well-spaced subset in $\mathrm{P}$ is $\mathrm{P}$-compressible.

Proof: Let $A \in \mathrm{E}$ and $B \in P$ be a well-spaced subset of $A$. If $A \neq \Sigma^{*}$, the following function $f$ is a polynomial-time minimal perfect hash function for $A$. For $x \notin B, f(x)=x$. For $x \in B, f(x)$ is the least $y \in B \cup \bar{A}$ so that $|y| \leq \log \log |x|$ and $y \neq f\left(x^{\prime}\right)$ for any $x^{\prime}<x$ in $B$. If no such $y$ exists, then $f(x)=x$.

By the definition of well-spaced sets, and the fact that $B \in \mathrm{P}$, for any $x$ in $B$, the set of $x^{\prime}<x$ in $B$ can be enumerated in polynomial time. Since $A \in \mathrm{DTIME}\left[2^{\text {cn }}\right], A$ or $\bar{A}$ can be enumerated up to length $\log (|x|)$ in polynomial time. Once these two lists are enumerated, computing $f\left(x^{\prime}\right)$ for $x^{\prime} \leq x$ in $B$ is quick. Note that $f$ is one-to-one, and $\bar{A} \cup B \subseteq f(B)$ and $A-B \subseteq f(A-B)$. Indeed, $f$ is a polynomial-time minimal perfect hash function for $A$.

Corollary 3.2 Every set in E that has an infinite P-printable subset is P-compressible.

Indeed, one can similarly show, using very widely spaced subsets of P-printable sets, that every recursive set that has an infinite $\mathrm{P}$-printable subset is $\mathrm{P}$-compressible. 
Corollary 3.3 $S A T$ is P-compressible.

A set $A$ is a cylinder if $A \equiv_{\text {iso }} A \times \Sigma^{*}$ via a recursive isomorphism. If the isomorphism is polynomial-time computable, then $A$ is a $\mathrm{P}$-cylinder [BH77,MY85]. If $A$ is $\mathrm{P}$-compressible and $B \equiv_{i s o}^{p} A$, then $B$ is also P-compressible. In particular, (1) all standard NP-complete sets (i.e., those of [GJ79]) are P-compressible (however, see [JY85,HHSY] for treatments of non-standard sets), and (2) if the Berman-Hartmanis conjecture (that all NP-complete sets are polynomial-time isomorphic to SAT) holds [BH77], then all NP-complete sets are P-compressible.

We have the following partial converse to Corollary 3.2.

Theorem 3.4 If $\mathrm{P}=\mathrm{UP}$ and $A \in \mathrm{P}$ is $\mathrm{P}$-compressible, then $A$ has an infinite $\mathrm{P}$-printable subset.

Proof: Let $f$ be a polynomial-time minimal perfect hash function for set $A \in \mathrm{P}$. The set:

$$
S=\left\{1^{n} \# y \# p \mid(\exists x \in A)[|x| \leq n, f(x)=y \text {, and } p \text { is a prefix of } x]\right\}
$$

is in UP. If $\mathrm{P}=\mathrm{UP}$, then $S \in \mathrm{P}$ can be used to invert $f$ on $A$ in polynomial time relative to the size of the inverse. Thus, $A \cap f^{-1}\left(0^{*}\right)$ is an infinite P-printable subset of $A$.

The following proposition is due to Russo (see [AR88]).

Proposition 3.5 Every infinite set in $\mathrm{P}$ has an infinite $\mathrm{P}$-printable subset if and only if every infinite set in NP has an infinite P-printable subset.

Notice that from Proposition 3.5 it follows that if all infinite sets in coNP have infinite P-printable subsets then all infinite sets in NP have infinite P-printable subsets.

Lemma 3.6 Every infinite set in $P$ has an infinite sparse coNP subset. Every infinite set in NP has an infinite sparse $\mathrm{D}^{\mathrm{P}}$ subset.

Proof: Recall that a set $C$ is in $\mathrm{D}^{\mathrm{P}}$ if and only if there are sets $A$ and $B$ in NP such that $C=$ $A-B$ [PY84]. Let $A$ be an infinite set in NP, and $B=\left\{x \in A \mid(\exists y \in A)\left[y<_{\text {lex }} x\right.\right.$ and $\left.\left.|x|=|y|\right]\right\}$, where <lex is the lexicographic order. Clearly, $B \in \mathrm{NP}$. Let $C=A-B$. Then $C \subseteq A$ is infinite if $A$ is, and, for each $n, C$ contains at most one string of length $n$. The other part of the claim is similarly direct.

It follows from the proof of Lemma 2.1 of [HY84] that if $E=\Sigma_{2}^{E}$ then all sparse $\mathrm{D}^{\mathrm{P}}$ sets are P-printable. From this, Lemma 3.6, and Corollary 3.2, it follows that:

Theorem 3.7 If $\mathrm{E}=\Sigma_{2}^{E}$ then every infinite set in NP is P-compressible.

Indeed, if $A \in \Sigma_{k+1}^{p}$, then $A$ has a sparse subset of the form $A-B$, with $A, B \in \Sigma_{k+1}^{p}$. Thus, via a natural generalization of the techniques of [HY84], if $E=E H$, every infinite set in $\Sigma_{k+1}^{p}$ has an infinite P-printable subset. Thus:

Corollary 3.8 If $\mathrm{E}=\mathrm{EH}$, then every infinite set in the polynomial hierarchy is $\mathrm{P}$-compressible.

Theorem 3.9 There is a recursive set $A$ that is $\mathrm{P}$-compressible, yet has no infinite P-printable subset. 
Proof: A recursive set $A^{\prime}$ can be constructed by diagonalization so that both $A^{\prime}$ and $\overline{A^{\prime}}$ intersect each infinite P-printable set infinitely often. Thus, neither $A^{\prime}$ nor $\overline{A^{\prime}}$ has an infinite P-printable subset. Therefore, $A=\left\{0 x \mid x \in A^{\prime}\right\} \cup\left\{1 x \mid x \in \overline{A^{\prime}}\right\}$ does not either. $f(i w)=w$ is a polynomial-time minimal perfect hash function for $A$, where $i \in\{0,1\}$.

Theorem 3.7 gives a sufficient condition- $\mathrm{E}=\Sigma_{2}^{E}$-for all infinite sets in NP to be P-compressible. However, this may not be a necessary condition. There is an oracle for which the conditions of Theorem 3.7 fail badly, yet not all infinite $\mathrm{NP}^{A}$ sets are $\mathrm{P}^{\boldsymbol{A}}$-compressible.

Theorem 3.10 extends, and the proof is in part inspired by, the famous result of Homer and Maass [HM83] stating that there is a relativized world in which $P$ differs from NP, yet all infinite NP sets have infinite subsets in P. Our result strengthens theirs in three ways: we achieve a stronger separation (NE versus coNE rather than NP versus $P$ ), our result applies to sets not only from NP but even from PSPACE, and, most crucially, our subsets are not only in $\mathrm{P}$ but are $\mathrm{P}$-printable.

Theorem 3.10 There exists a recursive oracle $A$ such that there is a tally set in $\mathrm{NP}^{A}-\operatorname{coNP}^{A}$, and each infinite set in PSPACE ${ }^{A}$ has an infinite $\mathrm{P}^{\boldsymbol{A}}$-printable subset.

Corollary 3.11 There exists an oracle $A$ such that $\mathrm{NE}^{A} \neq$ coNE $^{A}$, yet all infinite sets in PSPACE ${ }^{A}$ are $\mathbf{P}^{A}$-compressible.

Proof of Theorem 3.10 Let the $Q_{i}$ enumerate the NP oracle machines, with time-bound $q_{i}$, and let the $R_{i}$ enumerate the PSPACE oracle machines, with space-bound $q_{i}$. Recall that w.l.o.g. $q_{i}(r)=r^{i}+i$. We adopt the standard model of relativized PSPACE: the space bound is enforced on the oracle tape as well as the work tape.

$A$ will contain two kinds of strings. Let projection strings be strings of the form $1^{r} \alpha$, where $|\alpha|=r$. These are projected to form:

$$
H^{A}=\left\{1^{r} \mid(\exists \alpha)\left[|\alpha|=r \wedge 1^{r} \alpha \in A\right]\right\} .
$$

Clearly, $H^{A}$ is a sparse set in $N P^{A}$. Let coding strings be strings of the form $01^{i} 01^{q_{i}(r)} 0 \alpha$, where $|\alpha| \leq r$, for any string $\alpha$ and integer $r$. These encode the set

$$
S_{i}^{A}=\left\{\beta \mid 01^{i} 01^{q_{i}(|\beta|)} 0 \beta \in A\right\} .
$$

Our $A$ will be constructed so that the $S_{i}^{A}$ will be $P^{A}$-printable, and an infinite subset of $R_{i}^{A}$ in the case that $R_{i}^{A}$ is infinite. The $1^{q_{i}(r)}$ is padding, so if $|\beta|=r$, we are encoding " $\beta \in S_{i}^{A}$ " by using strings longer than any looked at in deciding whether $\beta \in R_{i}^{A}$.

Call $A$ legal if and only if:

1. A contains only projection and coding strings.

2. For each $i, r$ : if $01^{i} 01^{q_{i}(r)} 0 \alpha \in A$, and $\delta$ is any initial segment of $\alpha$, then $01^{i} 01^{q_{i}(r)} 0 \delta \in A$.

3. For each $i, r$ : if $01^{i} 01^{q_{i}(r)} 0 \alpha \in A$, then at most one of $01^{i} 01^{q_{i}(r)} 0 \alpha 0$ and $01^{i} 01^{q_{i}(r)} 0 \alpha 1$ is in $A$.

4. For each $i: S_{i}^{A} \subseteq R_{i}^{A}$.

This definition formalizes the "easy" part of the construction. If $A$ is legal, then each $S_{i}^{A}$ will be $P^{A}$-printable, since items $(2,3)$ say that for each $r, A$ encodes strings that give instructions for 
printing the unique $\beta$ (if there is any at all) of length $r$ with $01^{i} 01^{q_{i}(r)} 0 \beta \in A$. But, $S_{i}^{A}$ could be finite or even empty. In fact, $\emptyset$ is legal.

$A$ will be constructed in stages, and at each stage we will know $A$ up to strings of some length, $m$. If $A \subseteq \Sigma^{\leq m}$, say that $(A, m)$ is legal if and only if items $(1,2,3)$ above hold, and item (4) holds in the strong sense - namely, if $|\beta|=r$ and $\beta \in S_{i}^{A}$, then $\beta \in R_{i}^{A}$ and $q_{i}(r) \leq m$, so that extending $A$ to decide strings longer than $m$ will not change the acceptance of $\beta$ into $R_{i}^{A}$.

If $A \subseteq \Sigma^{\leq m}$ and $B \subseteq \Sigma^{\leq n}$, we say that $(B, n)$ extends $(A, m)$ if and only if $m \leq n, A \subseteq B$, and all strings in $B-A$ have length greater than $m$; i.e., we are viewing $(A, m)$ as a commitment to exclude all strings of length $\leq m$ not already in $A$.

At each stage in the construction we will do two things, so that we are constructing a sequence of numbers $0=n_{0}<m_{1}<n_{1}<m_{2}<n_{2}<\ldots$, and, for each $s, A_{s} \subseteq \Sigma \leq m$, and $B_{s} \subseteq \Sigma \leq n_{\text {. }}$. We can start with $B_{0}=\emptyset$. At stage $s$, we assume we have $n_{s}$ and $B_{s}$; we first (Step 1 ) choose $m_{s+1}$ and form $A_{s+1}$, with $\left(A_{s+1}, m_{s+1}\right)$ extending $\left(B_{s}, n_{s}\right)$; then (Step 2$)$ we choose $n_{s+1}$ and form $B_{s+1}$, with $\left(B_{s+1}, n_{s+1}\right)$ extending $\left(A_{s+1}, m_{s+1}\right)$. Each $\left(A_{s}, m_{s}\right)$ and $\left(B_{s}, n_{s}\right)$ will be legal, so

$$
A=\bigcup_{s=0}^{\infty} A_{s}=\bigcup_{s=0}^{\infty} B_{s}
$$

will be legal also. In particular, $S_{i}^{A}$ will be a subset of $R_{i}^{A}$, so we can now concentrate on making it infinite.

Before beginning the construction, let $\left\{i_{s} \mid s \geq 0\right\}$ list all natural numbers, with each number being repeated infinitely often. So, at stage $s$, if $i=i_{s}$ : Step 1 will be concerned with adding a new element of $S_{i}^{A}$; the fact that $i$ gets considered infinitely often will give us an opportunity to make $S_{i}^{A}$ be infinite. Step 2 will be concerned with making sure that $Q_{0}^{A}$ doesn't compute the complement of $H^{A}$; this need only be done once for each s.

Now, assume we have $n_{s}$ and $B_{s}$. We conclude the proof by describing the two steps and explaining why they ensure that the resulting $A$ satisfies the theorem.

Step 1: Set $i=i_{8}$. There are two cases:

Case 1.1: It is possible to choose an $r>n_{s}$, a string $\beta$ of length $r$, some $q \geq q_{i}(r)$, and some $C \subseteq \Sigma \leq q$ such that $(C, q)$ is legal, $\beta \in R_{i}^{C}$, and $(C, q)$ extends $\left(B_{s}, n_{s}\right)$. By truncating $C$, we may assume that $q$ is exactly $q_{i}(r)$, since longer strings don't figure in the acceptance of $\beta$. Now, we just extend $C$ enough to put $\beta$ into $S_{i}^{A}$. More formally, $m_{t+1}$ is $1+i+1+q+1+r$, and $A_{t+1}$ is $C$ together with all strings of the form $01^{i} 01^{\rho} 0 \delta$, where $\delta$ is any initial segment of $\beta$.

Case 1.2: Suppose Case 1.1 did not hold. Then let $m_{s+1}=n_{s}+1$ and let $A_{s+1}=B_{8}$.

Note: For a given $i$ : If case 1.2 ever happens at a stage $s$ with $i_{s}=i$, then $R_{i}^{A}$ will be finite, since $A$ itself is a legal extension of $\left(B_{s}, n_{8}\right)$, so that $R_{i}^{A}$ will contain only strings of length $\leq n_{8}$. However, if case 1.1 always happens, then $S_{i}^{A}$ will be infinite.

Step 2: Fix $r>m_{s+1}$ such that $q_{s}(r)<2^{r}$, and set $n_{s+1}=q_{s}(r)$. Let $D=A_{s+1}$, which we now consider as a subset of $\Sigma^{\Sigma_{r+1}}$. Again, there are two cases:

Case 2.1: $Q_{s}^{D}$ rejects $1^{r}$. Then set $B_{s+1}=D$. This ensures that $1^{r}$ will be in neither $H^{A}$ nor in $Q_{s}^{A}$.

Case 2.2: $Q_{s}^{D}$ accepts $1^{r}$. Fix an accepting computation, and let $W$ be the set of ocle strings queried during this computation. Let $B_{s+1}$ be $A_{s} \cup\left\{1^{r} \alpha\left|1^{r} \alpha \notin W \wedge\right| \alpha \mid=r\right\}$. Since $\|W\| \leq$ $q_{s}(r)<2^{r}, 1^{r}$ will be in $H^{A}$. Since we haven't changed the oracle on $W$, we will have $1^{r}$ in $Q_{s}^{A}$. 
Note: In either case, we have ensured that $H^{A}$ and $Q_{z}^{A}$ will not be complementary.

Note that the oracle constructed above will not be recursive, since at each step, deciding which of Cases 1.1 or 1.2 holds requires an infinite search. However, we can modify the proof to make the oracle recursive by the following priority argument.

We make some minor changes to simplify the notation and the arithmetic. We assume that the $R_{i}$ are enumerated so that $R_{0}^{A}$ always accepts all strings. Also, we may assume that each time and space bound, $q_{i}(r)$, satisfies $r<r^{\prime} \Rightarrow r<q_{i}(r)<q_{i}\left(r^{\prime}\right)$. Let $f(m, s)$ be the least $r>m$ such that $q_{s}(r)<2^{r}$, and let $g$ be a recursive function such that $g(m, s) \geq q_{s}(f(m, s))$ and $m<m^{\prime} \Rightarrow m<g(m, s)<g\left(m^{\prime}, s\right)$.

Step 2 of our construction is the same, except that we now specify that we take $r=f\left(m_{s+1}, s\right)$, and then $n_{s+1}=g\left(m_{s+1}, s\right)$.

We now describe our modified Step 1. Again, we have $n_{s}$ and $B_{s}$ and we want to find $m_{s+1}$ and $A_{s+1}$, but now we want our search to be effective.

First, some informal remarks: Clearly, we must replace the unbounded search for a $(C, q)$ in Step 1 by a bounded search - say, $q \leq w_{s}$. However, bounding the search demands some additional complexity in the construction. Consider a fixed index, $i$. At stages $s$ with $i_{s}=i$, we are trying to expand $R_{i}^{A}$ if possible - then we add appropriate coding strings for a new element of $S_{i_{0}}^{A}$. With the unbounded search, if such an expansion was impossible, we could permanently forget about $i$, but now, it might well be that $R_{i}^{A}$ could be expanded at a later stage. Perhaps in stages where $i_{s} \neq i$, we in fact expand $R_{i}^{A}$ "by accident" in the process of extending $A$ - then we don't add a coding string for $S_{i}^{A}$. If this happens too often, we might wind up with $R_{i}^{A}$ infinite but $S_{i}^{A}$ finite. It follows that we cannot actually choose the indices $i_{s}$ in advance. Instead, when we get to stage $s$, we choose $i$, from among the indices $\{0, \ldots, s\}$; in our choice, smaller $\left\|S_{i}^{B}\right\|$ get higher priority. The hope is that by continuing to focus on the smallest $\left\|S_{i}^{B}\right\|$ at each stage $s$, until we get to expand it, we will eventually make all $S_{i}^{A}$ infinite unless the corresponding $R_{i}^{A}$ is finite. However, we now observe that at stage $s$, we cannot actually work with a fixed bound $w_{s}>n_{s}$ depending only on $s$, where $w_{s}$ bounds the length of strings added to $A$ at this step. For example, say $S_{1}^{B}$ is empty, giving machine 1 high priority, but our search for a $(C, q)$ with $q \leq w_{s}$ is unable to expand $R_{1}^{A}$, so we pass on instead to machine number 2 ; we then add the appropriate coding string into $S_{2}^{A}$, and then proceed to Step 2, with $q=q_{2}(r)<m_{s+1}<n_{s+1}$. Now, $n_{s+1}$ or even $m_{s+1}$ could be larger than $w_{s}$, so it is quite possible that $\left(B_{s+1}, n_{s+1}\right)$ will put a new string into $R_{1}^{A}$, but it is already too late to code this string. If this same thing keeps happening, we might have $R_{1}^{A}$ infinite but $S_{1}^{A}$ empty. It follows that the bound $w$, must actually depend on $i$ as well, with higher priority indices having a higher bound; that is, if we decide to abandon machine 1 and take an expansion based on machine 2 and then use this to determine $n_{s+1}$, then the bound for machine 1 should have been at least as big as this $n_{s+1}$, so that we will be sure that our final oracle $A$ will not add any new strings into $R_{1}^{A}$ of lengths $\leq n_{s+1}$; strings longer than $n_{s+1}$ will not be a problem, because we still have a chance to code them at stage $s+1$.

Now, the formal details: Our priority function, $h$, is defined by: $h(i, s)=\left\|S_{i}^{B \cdot}\right\|+i$. Smaller $h$ values mean higher priority. The " $+i$ ", which adds a prejudice against large $i$, will be seen to be important later. Let $j_{s}^{0}, j_{s}^{1}, \ldots, j_{s}^{s}$ enumerate $\{0,1, \ldots, s\}$, with $\mu<\nu \Rightarrow h\left(j_{s}^{\mu}, s\right) \leq h\left(j_{s}^{\nu}, s\right)$. Then, fix numbers:

$$
n_{s}+1=r_{s}^{s}<w_{s}^{s}<r_{s}^{s-1}<\cdots<r_{s}^{0}<w_{s}^{0} .
$$


These are defined by saying $w_{s}^{\mu}=q_{j_{\mu}}\left(r_{s}^{\mu}\right)$, and, if $\mu>0$,

$$
r_{s}^{\mu-1}=g\left(1+j_{s}^{\mu}+1+q_{j \mu}^{\mu}\left(r_{s}^{\mu}\right)+1+r_{s}^{\mu}, s\right) .
$$

Each $r_{s}^{\mu}$ is an upper bound on the length of the sequence we are looking for if we decide to choose $j_{s}^{\mu}$, and $w_{s}^{\mu}$ is how far out we must extend our approximation to $A$ to be sure of the computation of this sequence. Next, let $\mu_{s}$ be the least $\mu$ such that there exists $\beta, D$ such that: $n_{s}<|\beta| \leq r_{s}^{\mu}$, $D \subseteq \Sigma^{w_{a}^{\mu}},\left(D, w_{s}^{\mu}\right)$ is legal, $\beta \in R_{j_{s}^{\mu}}^{D}$, and $\left(D, w_{s}^{\mu}\right)$ extends $\left(B_{s}, n_{s}\right)$. Note that there always at least one such $\mu$ - namely, with $j_{s}^{\mu}=0$, by our assumption that $R_{0}^{A}$ accepts all strings. Then, let $i_{s}$ be this $j_{s}^{\mu_{s}}$. If $q=q_{i}(|\beta|)$, then $|\beta|<q \leq w_{s}^{\mu_{1}}$; we set $C=D \cap \Sigma^{\leq q}$, and then determine $A_{s+1}$ and $m_{s+1}$ as in the old Step 1 by adding coding strings for $\beta \in S_{i}^{A}$. We then proceed with Step 2 , so that

$$
n_{s+1}=g\left(m_{s+1}, s\right)=g\left(1+i_{s}+1+q+1+|\beta|, s\right) \leq r_{s}^{\mu-1} .
$$

What our definition of the $r_{s}^{\mu}$ has accomplished is that if $\nu<\mu_{s}$, then $r_{s}^{\nu} \geq n_{s+1}$. Hence,

Observe: At each stage $s$ : if $i \leq s, A$ is any legal set of strings with $A \cap \Sigma^{n_{s}}=B_{s}$, and $\beta \in R_{i}^{A}$ with $n_{s}<|\beta| \leq n_{s+1}$, then $h\left(i_{s}, s\right) \leq h(i, s)$. If not, we would have had $i=j_{s}^{\nu}$ for some $\nu<\mu_{s}$, and we would not have passed over $\nu$ in choosing $\mu_{s}$.

This completes the construction. We must now check that it works; namely, that $S_{i}^{A}$ is infinite whenever $R_{i}^{A}$ is infinite. Suppose not. Let $b$ be the minimum among all finite $\left\|S_{i}^{A}\right\|+i$ such that $R_{i}^{A}$ is infinite. Let $i$ be any index with $\left\|S_{i}^{A}\right\|+i=b$ and $R_{i}^{A}$ infinite. Let stage $t \geq i$ be so that for all $j \leq b$ : if $S_{j}^{A}$ is finite, then $S_{j}^{A}=S_{j}^{B_{i}}$ and if $S_{j}^{A}$ is infinite, then $\left\|S_{j}^{B_{i}}\right\|>b$. Next, fix any string $\beta \in A$ with $|\beta|>n_{t}$. Finally, fix $s \geq t$ with $n_{s}<|\beta| \leq n_{s+1}$. By the above observation, $h\left(i_{s}, s\right) \leq h(i, s)$, so $\left\|S_{i,}^{B}\right\|+i_{s} \leq\left\|S_{i}^{B}\right\|+\bar{i}=\left\|S_{i}^{A}\right\|+i=b$. In particular, $i_{s} \leq b$, so $i_{s}$ is one of the $j$ looked at in defining $t$. Then $S_{i}^{A}$ cannot be infinite, since that would imply $\left\|S_{i}^{B}:\right\|>b$. But it also cannot be finite, since that would imply $S_{i}^{A}=S_{i,}^{B}$, but in fact $S_{i}^{A}$, always contains a new string of length $>n$, added at stage $s$.

We see now the importance of the " $+i$ " in the definition of the priority function $h$. The exact form of $h$ is not actually critical in this argument, but we could not have used just $\left\|S_{i}^{B}\right\|$, since we needed to have a fixed finite number of $j$ values to consider in defining the stage $t$.

Note that the proof of Theorem 3.10, and the techniques of that proof, establish claims stronger than those made in Theorem 3.10. In particular, oracle $A$ separates $\mathrm{NP}^{A}$ from the query class $\mathrm{QcoNP}^{A}$, and every set in QPSPACE ${ }^{A}$ has a $\mathrm{P}^{A}$-printable subset, where QcoNP and QPSPACE are the query classes of Tardos ([Tar87], see also [Boo81,BW81]). Similarly, with care one can stretch the time bounds on the diagonalization to replace coNP ${ }^{A}$ with some larger coNTIME $E^{A}$ classes.

Corollary 3.2 tells us that all infinite sets in $\mathrm{E}$ with infinite P-printable subsets are P-compressible. Does the converse hold? Although we cannot show that all P-compressible sets have infinite Pprintable subsets, we observe:

Theorem 3.12 If class $\mathcal{C}$ closed under intersection with $\mathrm{P}$ sets and $A \in \mathcal{C}$ is $\mathrm{P}$-compressible via some function $f$, then $A$ has an infinite sparse subset in $\mathcal{C}$, namely $f^{-1}\left(0^{*}\right) \cap A$.

Thus, to find an infinite set in NP that is not P-compressible, we need only find an infinite set in NP with no infinite sparse subsets in NP. It is not known whether such a set exists, but Theorem 3.13 constructs a relativized world in which one does. Combined with Lemma 3.6 , this 
gives optimal-with result to all relativizable proof techniques-bounds on the complexity of the easiest sparse subsets that $P$ sets perforce have (Corollary 3.14$){ }^{1}$

Theorem 3.13 There is an infinite set $A$ such that $A$ has no infinite sparse NP ${ }^{A}$ subsets. Therefore, neither $\mathrm{P}^{A}$ nor $\mathrm{NP}^{A}$ is $\mathrm{P}^{A}$-compressible.

Corollary 3.14 Though all infinite $\mathrm{P}$ sets have infinite sparse coNP subsets (this result holds also in all relativized worlds), there are relativized worlds in which some infinite $P$ sets have no infinite sparse NP subsets.

Proof of Theorem 3.13 Again, let the $Q_{i}$ enumerate all NP oracle machines, with $Q_{i}^{A}$ running with polynomial time bound $q_{i}$, regardless of the oracle. All we use about this time bound is that an accepting computation path of $Q_{i}^{A}$ that accepts a string $\alpha$ queries no more than $q_{i}(|\alpha|)$ oracle strings. For notational convenience, we will assume that $\alpha$ is always one of these strings.

Our construction will use a very widely spaced sequence of numbers, $n_{0}<n_{1}<n_{2}<n_{3}<\ldots$,

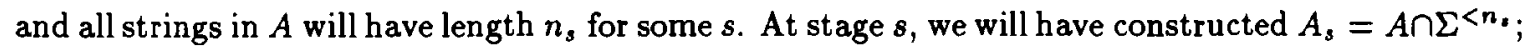
we will determine a set $K_{s} \subseteq \Sigma^{n}$, and then let $A_{s+1}=A_{s} \cup K_{s} . K_{s}$ will be chosen to ensure that for each $i<s, A \cap \Sigma^{n} \cdot \cap Q_{i}^{A}$ will be either empty or of size $\geq n^{s}$. Doing this for each $s$ will make each $A \cap Q_{i}^{A}$ either finite or non-sparse, so that $A$ will have no infinite sparse NP ${ }^{A}$ subsets. We always choose $n_{s+1}$ larger than $q_{i}\left(n_{s}\right)$ for all $i<s$, so that in constructing $K_{s}$, we will not have to worry about what strings will be put in later; whatever the final $A$, we will know that for $i<s, Q_{i}^{A}$ and $Q_{i}^{A,+1}$ will accept the same strings of length $n_{s}$.

We specify the $n_{s}$ in advance. We can start with $n_{0}=0$ and $A_{1}=\emptyset$, so that the first stage in the construction will be stage 1 , where we determine $K_{1}$ and $A_{2}$. For $s>0$, take $n_{s}$ to be any $n>n_{s-1}$ such that

a. For all $i<s, n>q_{i}\left(n_{s-1}\right)$, and

b. For all $i<s, 2^{n}>s \cdot q_{i}(s) \cdot\left[\left(s \cdot n^{s} \cdot q_{i}(s)+1\right) \cdot s \cdot s \cdot n^{s}\right]$.

Item (a) was explained above. The reason for the particular numerology in (b) will appear below. For now, it is enough to note that such an $n$ exists, since the expression that $2^{n}$ must dominate is bounded by a polynomial function of $n$.

${ }^{1}$ This touches upon a very interesting point. How hard is it to "thin down" a set? That is, given complexity classes $\mathcal{C}_{1}$ and $\mathcal{C}_{2}$, is it always the case that every infinite set from $\mathcal{C}_{1}$ has an infinite sparse subset in $\mathcal{C}_{2}$. Let's say that $\mathcal{C}_{1}$ is $\mathcal{C}_{2}$-sparse-immune if there is an infinite set in $\mathcal{C}_{1}$ that has no infinite sparse $\mathcal{C}_{2}$ subsets. This notion is akin to immunity. Though a slightly weaker separation than full immunity, sparse-immunity is of use in cases where full immunity results are precluded. For example, $\mathcal{C}_{1}$ is never $\mathcal{C}_{1}$-immune, but Theorem 3.13 shows that there is a relativized world in which $\mathrm{P}$ is $\mathrm{P}$-sparse-immune. This is a graphic illustration of the cost of choosing a sparse subset of a set. In the final version of this paper, we intend to explore sparse-immunity in detail. For now, we summarize our upper-bound results: $P$ is not coNP-sparse-immune; $N P$ is not $D^{P}$-sparse-immune; coNP is not $\Pi_{2}^{p}$-sparse-immune; relativizing these three results gives sparse-immunity results for $\Delta_{i}^{p}, \Sigma_{i}^{p}$, and $\Pi_{i}^{p}$; PSPACE is not PSPACE-sparse-immune; E is not E-sparse immune; NE is not $D^{E}$-sparse immune; coNE is not $P^{N E}$-sparse-immune. The surprisingly tight $\mathrm{P}^{N E}$ upper bound on the easiest infinite sparse subsets that coNE sets perforce have uses the fact that the natural sparseimmunity result would be coNPNE. However, this is a class in the strong exponential hierarchy, which is known to collapse [Hem89]. 
Now, say we are at stage $s$, and set $n=n_{s}$. So, we have already determined $A_{s}$. For any $X \subseteq \Sigma^{n}$, let $B_{X}=A_{s} \cup X$. The proof will be done when we explain how to find a $K \subseteq \Sigma^{n}$ such that $K \cap Q_{i}^{B_{K}}$ is either empty or of size $\geq n^{s}$ for all $i<s$; then this $K$ will be our $K_{s}$. Of course, $K$ will have to be non-empty, so that the resultant $A$ will be infinite.

First, let

$$
\begin{array}{cc}
v & =\max \left\{q_{i}(n) \mid i<s\right\} \\
r & =\left(s \cdot n^{s} \cdot v+1\right) \cdot s \\
u & =r \cdot s \cdot n^{s} \\
w & =v \cdot u .
\end{array}
$$

This numerology will also be explained as we progress.

Next, let's decide which of the $K \cap Q_{i}^{B_{K}}$ will be empty. Call an excluding pair a pair $(f, H)$, where $f \subseteq\{i \mid i<s\}, H \subseteq \Sigma^{n},\left\|\Sigma^{n}-H\right\| \leq\|f\| \cdot w$, and for all $X \subseteq H, X \cap Q_{i}^{B_{X}}=\emptyset$ for all $i \in f$. So, $\left(\emptyset, \Sigma^{n}\right)$ is an excluding pair. If $(f, H)$ is an excluding pair, then $H$ is non-empty, since $\left\|\Sigma^{n}-H\right\| \leq s \cdot w$ and our numerology implies that $2^{n}>s \cdot w$. We partially order the excluding pairs by saying $\left(f_{1}, H_{1}\right) \leq\left(f_{2}, H_{2}\right)$ if and only if $f_{1} \subseteq f_{2}$ and $H_{1} \supseteq H_{2}$ (we exclude more indices, but allow ourselves a slightly smaller $H$ to do the excluding). Now, fix an excluding pair $(f, H)$ which is maximal in this order. Our final $K$ will be a subset of this $H$, so we will certainly have $K \cap Q_{i}^{B_{K}}=\emptyset$ for all $i \in f$. For $i \notin f$, maximality (the fact that $i$ cannot be added to $f$ ) will aid us in making $K \cap Q_{i}^{B_{K}}$ big.

Let $g=\{i \mid i<s \wedge i \notin f\}$; these are the indices we have to worry about. If $g=\emptyset$, we can simply set $K=H$ (as we observed above, $H \neq \emptyset$ ), so we assume in what follows that $g$ is non-empty. Say $\|g\|=d$, and let $g=\left\{i_{0}, \ldots, i_{d-1}\right\}$. For any $k$, let $i_{k}=i_{k \text { modd }}$. We are planning an iterated construction in $u$ steps; at step $k$, we will look at machine $i_{k}$; so each $i \in g$ will get looked at $u / d$ times.

We will define a decreasing sequence of sets,

$$
H=L_{0} \supseteq L_{1} \supseteq L_{2} \supseteq \ldots L_{u} .
$$

For each $k$, we will choose a string $\alpha_{k} \in L_{k}$, and some sets $V_{k}, V_{k}^{+}, V_{k}^{-}$, and then, when $k+1 \leq u$, define $L_{k+1}$. We verify inductively that for each $k,\left\|H-L_{k}\right\| \leq k \cdot v$. First fix $i=i_{k}$. Maximality of $(f, H)$ implies that $\left(f \cup\{i\}, L_{k}\right)$ is not an excluding pair. Our numerology guarantees that $\left\|\Sigma^{n}-L_{k}\right\| \leq\|(f \cup\{i\})\| \cdot w$, since $\left\|\Sigma^{n}-H\right\| \leq\|f\| \cdot w$ and $\left\|H-L_{k}\right\| \leq k \cdot v \leq u \cdot v=w$. Thus, the other condition of "excluding pair" must fail, so we can find an $X \subseteq L_{k}$ and choose $\alpha_{k} \in X$ which is accepted by $Q_{i}^{B_{X}}$. Fix an accepting computation, and let $V_{k}$ be the set of oracle strings in $\Sigma_{n}$ which are queried in this computation; so $\alpha_{k} \in V_{k}$ and $\left\|V_{k}\right\| \leq v$. Let $V_{k}^{+}=V_{k} \cap X$ (these queries answered "yes"), and let $V_{k}^{-}=V_{k}-X$ (these queries answered "no"). If $k+1 \leq u$, set $L_{k+1}=L_{k}-V_{k}$. Observe:

$$
\alpha_{k} \in V_{k}^{+} \subseteq L_{k} \quad \& \quad j<k \Rightarrow V_{j} \cap L_{k}=\emptyset .
$$

In particular, the $\alpha_{k}$ are all distinct.

Whenever $Y \subseteq \Sigma^{n}$ is such that $V_{k}^{+} \subseteq Y$ and $V_{k}^{-} \cap Y=\emptyset, \alpha_{k} \in Y \cap Q_{i_{k}}^{B_{Y}}$. For any set $J$ of indices, let $K_{J}=\bigcup\left\{V_{k}^{+} \mid k \in J\right\}$. Call $J$ free if and only if whenever $j, k$ are distinct members of $J$, 
$V_{j}^{+} \cap V_{k}^{-}=V_{j}^{-} \cap V_{k}^{+}=\emptyset$. If $J$ is a free set of indices, then for each $k \in J, \alpha_{k} \in K_{J} \cap Q_{i}^{B_{K_{J}}}$. We are thus done if we can find such a free $J$ such that for each $i \in g,\left\{k \in J \mid i_{k}=i\right\}$ has size $n^{s}$.

If $j<k$, then, by $(*), V_{j}^{-} \cap V_{k}^{+}=\emptyset$; we thus need only be concerned with making $V_{j}^{+} \cap V_{k}^{-}=\emptyset$. Also by $(*)$, the $V_{j}^{+}$are all disjoint. Informally, if we fix a $k, V_{k}^{-}$is small, so there are not too many $j<k$ for which $V_{j}^{+}$can meet it. Formally, we choose $J$ top-down; $J$ will be $\left\{k_{\mu} \mid \mu<d \cdot n^{s}\right\}$, where $u=k_{0}>k_{1}>\ldots$ and $k_{\mu} \bmod d=\mu \bmod d$ for each $\mu$. We inductively verify that each $k_{\mu} \geq u-r \cdot \mu$. In particular, each $k_{\mu} \geq r$, since $\mu<d \cdot n^{s} \leq s \cdot n^{s}$, so $k_{\mu} \geq u-r \cdot\left(s \cdot n^{s}-1\right)$ and $u=r \cdot s \cdot n^{s}$. Given $k_{\mu}$, now, we are done if we can find $k=k_{\mu+1}$. Let $e=(\mu+1) \bmod d$. We need:

$$
\begin{aligned}
& k_{\mu}-r \leq k<k_{\mu} . \\
& V_{k}^{+} \cap V_{k_{\nu}}^{-}=\emptyset \text { for } \nu=0, \ldots, \mu .
\end{aligned}
$$$$
k \bmod d=e \text {. }
$$

Let $V=\bigcup_{\nu=0}^{\mu} V_{k_{\nu}}^{-}$. Then $\|V\| \leq(\mu+1) \cdot v \leq s \cdot n^{s} \cdot v$. Among the $r$ numbers, $\left\{k \mid k_{\mu}-r \leq k<k_{\mu}\right\}$, there are at least $\|V\|+1$ with $k \bmod d=e$, since our numerology gives $r=\left(s \cdot n^{0} \cdot v+1\right) \cdot s \geq$ $(\|V\|+1) \cdot d$. We may then choose such a $k$ with $V_{k}^{+} \cap V=\emptyset$; this is possible because the $V_{k}^{+}$are all disjoint.

Since the question of whether all infinite sets in NP are P-compressible can be relativized in both directions, as can the question of whether all infinite sets in $\mathrm{P}$ are $\mathrm{P}$-compressible, we conclude that the $\mathrm{P}$-compressibility of $\mathrm{P}$ and NP cannot be finally resolved by methods that relativize. However, this section has provided necessary structural conditions and sufficient structural conditions.

\subsection{Polynomially Honest Minimal Perfect Hash Functions}

Recall that a function $f: \Sigma^{*} \rightarrow \Sigma^{*}$ is polynomially honest if there is a polynomial function $h(n)$ such that for all $x \in \Sigma^{*},|x| \leq h(|f(x)|)$. Informally, a function is polynomially honest if it never shrinks its input overmuch. In this section, we discuss the complexity of sets having polynomially honest polynomial-time minimal perfect hash functions. In particular, we give evidence that complete sets in the polynomial hierarchy lack such functions.

Lemma 3.15 If $A \in \mathrm{NP}$ is honestly $\mathrm{P}$-compressible, then $A \in \mathrm{NP} \cap$ coNP.

Proof: Suppose $A$ has such a function $f$. Then the following gives an NP decision procedure for $\bar{A}$. Given $x$, guess $y \neq x$ such that $f(x)=f(y)$, and a certificate, $c$, for $y \in A$. The pair $\langle y, c\rangle$ forms a certificate for $x \in \bar{A}$.

A set $A$ is in $H_{k}(h i g h-k)$ [Sch83] if $A \in \mathrm{NP}$ and $\Sigma_{k}^{p, A}=\Sigma_{k+1}^{p}$.

Theorem 3.16 If any set in $H_{k}$ has an honest polynomial-time minimal perfect hash function, then $\mathrm{PH}=\Sigma_{k}^{p}$.

Corollary 3.17 1. If any $\leq_{S N^{-}}^{p}$-complete set for NP is honestly $\mathrm{P}$-compressible, then NP $=$ coNP.

2. If any $\leq_{T}^{p}$-complete set for NP has an honest minimal perfect hash function computable in $P$ (or even $\mathrm{NP}_{s v}$, tot), then $\mathrm{NP}=$ coNP.

These results relativize to produce analogous results for higher levels of the polynomial hierarchy.

Finally, we look at polynomially honest polynomial-time minimal perfect hash functions on sets in $\mathrm{P}$. 
Proposition 3.18 If $\mathrm{P}=\mathrm{UP}, A$ and $B \in \mathrm{P}$, and $A, \bar{A}, B$, and $\bar{B}$ are honestly $\mathrm{P}$-compressible, then $A \equiv_{i s o}^{p} B$.

Proof: Let $f_{1}$ and $f_{2}$ be honest polynomial-time computable minimal perfect hash functions for $A$ and $\bar{A}$, respectively. Define:

$$
f(x)= \begin{cases}0 f_{1}(x) & x \in A \\ 1 f_{2}(x) & x \in \bar{A}\end{cases}
$$

$f$ is one-to-one and polynomially honest, so $\mathrm{P}=\mathrm{UP}$ implies that it is invertible [GS88]. Define $g$ similarly on $B$ and $\bar{B}$. Since $f$ and $g$ are both onto $\Sigma^{*}$ and $f(A)=g(B)$, it follows that $h(x)=$ $f^{-1}(g(x))$ is the desired isomorphism.

\subsection{Compression versus Ranking}

In the introduction, we mentioned an apparently stronger form of minimal perfect hash functions, namely ranking [GS85,Huy88,HR90]. We now ask how polynomial-time ranking functions (see Section 2) compare with the seemingly more general notion of polynomial-time minimal perfect hash functions. Are the two notions equivalent or different for sets in P? That is, are there sets in P that have polynomial-time minimal perfect hash functions, but no polynomial-time ranking functions?

Theorem 3.19 There is a set $A \in \mathrm{P}$ that is not $\mathrm{P}$-rankable if and only if there is a set $B \in \mathrm{P}$ that is honestly P-compressible and not P-rankable.

Proof: Let $A \in \mathrm{P}$ be a set that is not P-rankable. Define $B=\{0 x \mid x \in A\} \cup\{1 x \mid x \in \bar{A}\}$, and let $f(i w)=w, i \in\{0,1\}$. Let $r_{A}$ and $r_{B}$ be the ranking functions of $A$ and $B$, respectively. Notice that $r_{B}\left(1^{n}\right)=2^{n}-1$ for all $n>0$. If $r_{B} \in \mathrm{P}$, we would have the following polynomial ranking function for $A$.

$$
r_{A}(w)=\sum_{j=1}^{w} \underbrace{\left(r_{B}\left(01^{j-1}\right)-r_{B}\left(1^{j-1}\right)\right)}_{\text {\# of strings of length } j-1 \text { in } A}+\underbrace{r_{B}(0 w)-2^{|w|}-1}_{\text {\# of strings of length }|w| \text { in } A}
$$

Furthermore, if we let $B^{\prime}=\{0 x \mid x \in B\} \cup\{1 x \mid x \in \bar{B}\}, B^{\prime}$ is still P-compressible, but is not P-rankable.

The converse is immediate.

It is known that $P \neq P \# P$ if and only if there are sets in $P$ that are not $P$-rankable [GS85,HR90]. Thus we may interpret Theorem 3.19 as saying that, unless the polynomial hierarchy collapses to $\mathrm{P}$, not all honestly $\mathrm{P}$-compressible sets in $\mathrm{P}$ are $\mathrm{P}$-rankable.

Corollary $3.20 \mathrm{P} \neq \mathrm{P \# P}$ if and only if there are sets in $\mathrm{P}$ that are $\mathrm{P}$-compressible but not $\mathrm{P}$ rankable.

Baker, Gill, and Solovay showed that $\mathrm{P}^{A} \neq N \mathrm{P}^{A}$ with probability one relative to a random oracle $A$ [BGS75]. Thus, since Corollary 3.20 relativizes:

Corollary 3.21 With probability one relative to a random oracle $A$, there are sets in $\mathrm{P}^{A}$ that are $\mathrm{P}^{A}$-compressible but not $\mathrm{P}^{A}$-rankable. 


\section{Conclusions}

This paper studied the complexity of P-compressible sets, and proved both necessary structural conditions and sufficient structural conditions to ensure that all infinite NP sets are P-compressible. It remains an open question whether there is a single set of structural conditions that completely characterizes the question of whether all infinite NP sets are P-compressible. In the process of establishing our results, we proved essentially optimal-with respect to relativizable proof techniquesbounds on the complexity of sparse subsets of $P$ sets: though all infinite $P$ sets have infinite sparse coNP subsets, in relativized worlds some infinite $P$ sets have no infinite sparse NP subsets.

Finally, though this paper has focused on the study of polynomial-time minimal perfect hash functions, one could also study recursion-theoretic minimal perfect hash functions. The results one gets in the crisp setting of recursive function theory are loosely analogous to the results of this paper, but, as is usually the case, are far more exact and are easily obtained: An infinite RE set $A$ is recursive if and only if it has a recursive minimal perfect hash function; all coRE-complete sets have recursive minimal perfect hash functions, however no recursive minimal perfect hash function for any coRE-complete set is recursively honest.

\section{Acknowledgements}

We would like to thank Richard Karp for the discussions that originally inspired this work.

\section{References}

[Ad179] L. Adleman. Time, space, and randomness. Technical Report MIT/LCS/TM-131, MIT, Cambridge, MA, 1979.

[AL88] A. Aho and D. Lee. Storing a dynamic sparse table. In Proceedings 27th IEEE Symposium on Foundations of Computer Science, pages 55-60. IEEE Computer Society Press, 1988.

[AR88] E. Allender and R. Rubinstein. P-printable sets. SIAM Journal on Computing, 17(6):1193-1202, 1988.

[BGS75] T. Baker, J. Gill, and R. Solovay. Relativizations of the P=?NP question. SIA M Journal on Computing, 4(4):431-442, 1975.

[BH77] L. Berman and J. Hartmanis. On isomorphisms and density of NP and other complete sets. SIAM Journal on Computing, 6(2):305-322, 1977.

[Boo81] R. Book. Bounded query machines: On NP and PSPACE. Theoretical Computer Science, 15:27-39, 1981 .

[BW81] R. Book and C. Wrathall. Bounded query machines: On NP( ) and NPQUERY( ). Theoretical Computer Science, 15:41-50, 1981.

[CC88] C. Chang and C. Chang. An ordered minimal perfect hashing scheme with one parameter. Information Processing Letters, 27:79-83, 1988.

[Cha84] C. Chang. The study of an ordered minimal perfect hashing. Communications of the $A C M, 27(4): 384-387,1984$. 
[DKM $\left.{ }^{+} 88\right]$ M. Dietzfelbinger, A. Karlin, K. Mehlhorn, F. Meyer auf der Heide, H. Rohnert, and R. Tarjan. Dynamic perfect hashing: Upper and lower bounds. In Proceedings 29th IEEE Symposium on Foundations of Computer Science, pages 524-531. IEEE Computer Society Press, 1988.

[GJ79] M. Garey and D. Johnson. Computers and Intractability: A Guide to the Theory of NP-Completes. W. H. Freeman and Company, 1979.

[Gor89] S. Gordon. A mathematical formulation of the requirements of an order preserving hash function. In Proceedings of the International Conference on Computing and Information, pages 64-68. Canadian Scholars' Press Inc., 1989.

[GS85] A. Goldberg and M. Sipser. Compression and ranking. In 17th ACM Symposium on Theory of Computing, pages 440-448, 1985.

[GS88] J. Grollmann and A. Selman. Complexity measures for public-key cryptosystems. SIAM Journal on Computing, 17:309-335, 1988.

[Hem89] L. Hemachandra. The strong exponential hierarchy collapses. Journal of Computer and System Sciences, 39(3):299-322, 1989.

[Hem90] L. Hemachandra. Algorithms from complexity theory: Polynomial-time operations for complex sets. In Proceedings SIGAL International Symposium on Algorithms. SpringerVerlag Lecture Notes in Computer Science, 1990. To appear.

[HHSY] L. Hemachandra, A. Hoene, D. Siefkes, and P. Young. On sets polynomially enumerable by iteration. Theoretical Computer Science. To appear.

[HIS85] J. Hartmanis, N. Immerman, and V. Sewelson. Sparse sets in NP-P: EXPTIME versus NEXPTIME. Information and Control, 65(2/3):159-181, May/June 1985.

[HM83] S. Homer and W. Maass. Oracle dependent properties of the lattice of NP sets. Theoretical Computer Science, 24:279-289, 1983.

[HR90] L. Hemachandra and S. Rudich. On ranking. Journal of Computer and System Sciences, 40, 1990. To appear.

[Huy88] D. Huynh. The complexity of ranking. In Proceedings Srd Structure in Complexity Theory Conference, pages 204-212. IEEE Computer Society Press, June 1988.

[HY84] J. Hartmanis and Y. Yesha. Computation times of NP sets of different densities. Theoretical Computer Science, 34:17-32, 1984.

[JY85] D. Joseph and P. Young. Some remarks on witness functions for non-polynomial and non-complete sets in NP. Theoretical Computer Science, 39:225-237, 1985.

[Knu73] D. Knuth. The Art of Computer Programming: Sorting and Searching, volume 3 of Computer Science and Information. Addison-Wesley, 1973.

[Mah86] S. Mahaney. Sparse sets and reducibilities. In R. Book, editor, Studies in Complexity Theory, pages 63-118. John Wiley and Sons, 1986.

[MNT90] Y. Mansour, N. Nisan, and P. Tiwari. The computational complexity of universal hashing. In Proceedings 22nd ACM Symposium on Theory of Computing, 1990. To appear.

[MY85] S. Mahaney and P. Young. Reductions among polynomial isomorphism types. Theoretical Computer Science, 39:207-224, 1985. 
[PY84] C. Papadimitriou and M. Yannakakis. The complexity of facets (and some facets of complexity). Journal of Computer and System Sciences, 28(2):244-259, 1984.

[Sch83] U. Schöning. A low and a high hierarchy in NP. Journal of Computer and System Sciences., 27:14-28, 1983.

[Spr77] R. Sprugnoli. Perfect hashing functions: A single probe retrieving method for static sets. Communications of the $A C M, 20(11): 841-850,1977$.

[Tar87] G. Tardos. Query complexity, or why is it difficult to separate NPA $\cap$ coNPA from $\mathrm{P}^{A}$ by random oracles $A$. Manuscript, July 1987.

[Val76] L. Valiant. The relative complexity of checking and evaluating. Information Processing Letters, 5:20-23, 1976.

[Val79] L. Valiant. The complexity of enumeration and reliability problems. SIAM Journal on Computing, 8(3):410-421, 1.979. 